\title{
Device to Device Hybridization in 5G and Beyond: A Literature Review
}

\author{
${ }^{* 1}$ Faisal Lawal, ${ }^{1}$ Aliyu D. Usman, ${ }^{1}$ Abdoulie M.S. Tekanyi, ${ }^{1}$ Hassan A. Abdulkarim and ${ }^{2}$ Abubakar L. Tanko \\ ${ }^{1}$ Department of Electronics and Telecommunications Engineering, Ahmadu Bello University, Zaria, Nigeria \\ ${ }^{2}$ School of Information Technology and Computing, American University of Nigeria, Yola, Nigeria \\ \{flawa199 | aliyuusman1 | abubakarlamido\}@gmail.com I amtekanyi@abu.edu.ng | ha2zx@yahoo.com
}

\section{REVIEW ARTICLE}

Received: 14-OCT-2021; Reviewed: 18-NOV-2021; Accepted: 20-DEC-2021

http://dx.doi.org/10.46792/fuoyejet.v6i4.709

\begin{abstract}
Device-to-Device (D2D) communication is one of the most promising technologies to enhance user experience in 5G and beyond. Despite the huge benefit anticipated, enabling D2D in cellular network has encounter some challenges, these challenges include peer discovery and synchronization, mode selection and interference management. However, resolving these challenges promises improved service delivery, spectrum efficiency and reduced latency amongst other gains. Attempts to enable D2D in both microwave and millimetre wave network gained some traction in recent years in a bid to enable wider coverage and utilization of the technology. Some of the research attempts, challenges and prosects are discussed in this paper.
\end{abstract}

Keywords- Device-to-device, microwave, millimetre wave, inter-cell Interference

\section{INTRODUCTION}

The he most promising solution to spectrum scarcity is the incorporation of emerging technology into the next generation of cellular network. These emerging technologies has the potential to improve spectrum efficiency, reduce latency, and increase flexibility while also ensuring seamless transition from one cellular network generation to the next. One of the front runners of the technology to help in spectrum management and improve user experience is Device-to-Device D2D communication. D2D enabled cellular network is reported to suffer intercell interference (Kuruvatti, Hernandez, \& Schotten, 2019), thereby degrading cellular network user service quality (Noura \& Nordin, 2016).

With the rapid deployment of millimeter wave and the existing microwave technology D2D finds application in a hybrid environment where it can be deployed to serve both microwave and millimeter wave network users without significant change in infrastructure, while also providing means of interference mitigation. Attempts have been put forward by researchers to enable D2D communication in 4G microwave cellular networks (Lien, Chien, Tseng, \& Ho, 2016; Sanusi, Nasr, \& Moessner, 2019) also for exclusive 5G millimeter wave networks (Qiao et al., 2015; Ramadhan \& Nashiruddin, 2020; Sarma \& Hazra, 2020), recent research geared towards use of D2D technology in hybrid 4G and 5G networks (Hussain, Jangsher, Arafah, Mao, \& Li, 2019; Prado, Cardieri, \& Brito, 2020; Roslee, Nizam, \& Yusoff, 2020; Wang, Wang, Feng, \& Xu, 2019).

\footnotetext{
${ }^{*}$ Corresponding Author

Section B- ELECTRICAL/ COMPUTER ENGINEERING \& RELATED SCIENCES

Can be cited as:

Lawal F., Usman A.D., Tekanyi A.M.S., Abdulkarim H.A. and Tanko A.L. (2021): "Device to Device Hybridization in 5G and Beyond: A Literature Review", FUOYE Journal of Engineering and Technology (FUOYEJET), 6(4), 363368. http://dx.doi.org/10.46792/fuoyejet.v6i4.709
}

\section{Device-to-Device (D2D) Communication}

Device-to-device communication generally refers to a form of technology that makes possible nearby devices to communicate directly with each other over the licensed cellular bandwidth with limited or no permission from Access Point (AP) or Base Station (BS). D2D was proposed as part of 4G LTE-A network and was part of 3GPP LTEA standard in Release 12 as ProSe (Proximity Service) (Lien et al., 2016). With the proliferation of new contextaware services and applications, and new trend in cooperative communication paradigm shifting from fixed terminal relaying to device relaying, D2D communication is expected to continue to evolve and play an important role in future 5G networks (Höyhtyä, Apilo, \& Lasanen, 2018). D2D communication is broadly classified into Inband and outband communication, depicted in figure 1.
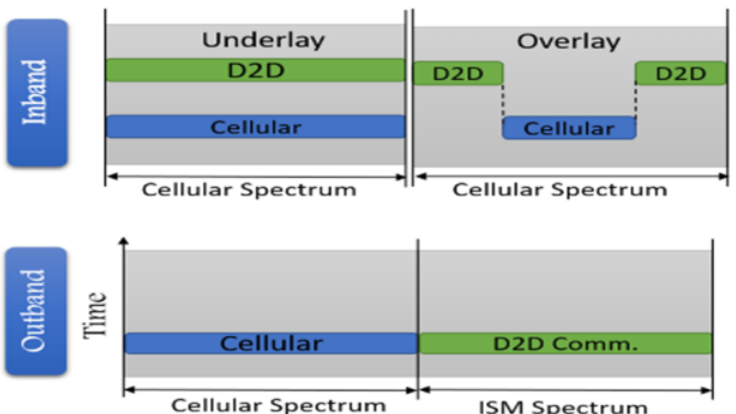

Fig.1: Inband and Outband D2D configurations (Asadi et al., 2014)

D2D communication operates in basically three different configuration which are either Network controlled, Autonomous or Network assisted D2D communication (Song, Niyato, Han, \& Hossain, 2015). Some of the major drawback of D2D communication are;

i. Interference Management: this is one of the most critical challenges of D2D as the interference between D2D equipment and cellular equipment. Several techniques have been proposed to mitigate interference amongst which is resource allocation technique (Dai \& Gui, 2020; Guizani \& Hamdi, 2016) and power control technique (Kuruvatti et al., 2019; Sanusi et al., 2019). 
ii. Mode Selection: Mode selection can be described as the process of determining whether the D2D pair should communicate in D2D mode or if a cellular mode (CM). This is vital for the successful seamless implementation of D2D in hybrid network and improve user satisfaction. D2D operating modes can be classified as;

a. Dedicated Mode - Here resources are assigned to D2D users, thereby preventing co-channel interference. Signal to Noise Ratio in DM mode is computed by;

$$
\gamma_{i}^{d m}=\frac{\rho_{i} G_{i}^{k}}{N_{0}}
$$

and the throughput is obtained by the expression;

$$
T_{i}^{d m}=w_{B} \log _{2}\left(1+\gamma_{i}^{d m}\right)
$$

where, $\rho_{i}$ is the power of D2D transmitter $D 2 D_{t x}$ of pair $i^{t h}$ and $G_{i}^{k}$ channel gain between $D 2 D_{t x}$ and $D 2 D_{r x}$.

b. Reuse Mode: for Reuse Mode, D2D users share the Cellular user equipment channel, this however causes interference for users in of both tiers. The SINR of the users $\{m, s, i\}$ under macro, small, and D2D tier communicating in the same Resource block (RB) $k^{\text {th }}$ is describe mathematical by;

$$
\gamma_{m}^{k}=\frac{P_{M B} G_{M B, m}^{k}}{N_{0}+\sum_{j=1}^{d} y_{j}^{k} h_{j, m}^{k} p_{j}+\sum_{j=1}^{N} Y_{S B_{j}}^{k} h_{S B_{j, m}}^{k} P_{S B_{j}}}
$$

where, $\left\{G_{M B, m}^{k}, G_{S B, m}^{k}, G_{i}^{k}\right\}$ represents the channel gain between $\mathrm{MB}$ and $m^{\text {th }}$ user.

\section{D2D Communication System Model}

Considering a sectored antenna array model, as reported in (Chevillon, Andrieux, Négrier, \& Diouris, 2018), for both transmitter and receiver in mmWave, where $\phi_{b}$ and $\phi_{u}$ denotes half-power beamwidth of BSs and UEs respectively. Main lobe and side-lobe gain are represented by $G m_{b}$ and $G s_{b}$ respectively. $G m_{u}$ and $G s_{u}$ denotes receiving UEs main and side lobe gains respectively. Furthermore, assuming an $N \times N$ uniform planar square antenna array, with half-wavelength antenna spacing, the beamwidth $\phi$, main lobe gain $G m$ and side lobe gain $G s$ are obtained as $(\mathrm{Wu}$, Atat, Mastronarde, \& Liu, 2018);

$$
\phi=\frac{1.732}{N}, G m=N^{2}, G s=\frac{1}{\sin ^{2}}\left(\frac{3 \pi}{2 N}\right)
$$

Antenna array gain $g_{i}$ from an arbitrary $B S i$ to a typical cellular receiver is represented as;

$g_{i}=$

$\left\{\begin{array}{l}G_{1}=G m_{b} G m_{u}, \text { with probability (w.p.) } \frac{\phi_{b}}{2 \pi} \frac{\phi_{u}}{2 \pi}, \\ G_{2}=G m_{b} G s_{u}, \text { with probability (w.p.) } \frac{\phi_{b}}{2 \pi}\left(1-\frac{\phi_{u}}{2 \pi}\right), \\ G_{3}=G s_{b} G m_{u} \text {, with probability (w.p.) }\left(1-\frac{\phi_{b}}{2 \pi}\right) \frac{\phi_{u}}{2 \pi}, \\ G_{4}=G s_{b} G m_{u}, \text { with probability (w.p.) }\left(1-\frac{\phi_{b}}{2 \pi}\right)\left(1-\frac{\phi_{u}}{2 \pi}\right)\end{array}\right.$

four possibly antenna gains are denoted for simplicity as $G_{k}=1,2,3,4$. where, $G_{1}=G m_{b} G m_{u}$ is the boresight gain (Wu et al., 2018).
Using Poison Point Distribution (PPP), the LOS probability of two nodes separated by a distance $d$ on the plane is obtained by (Wu et al., 2018);

$$
\rho L(d)=\exp \left(-\eta \lambda_{0}\left(2 E[R] d+\pi E\left[R^{2}\right]\right)\right)
$$

where $\lambda_{0}$ is the obstacle intensity, the random variable $R$ denotes the obstacle radius.

$$
\eta=1-\int_{0}^{1 \int} \int_{h_{\min }}^{s h_{R x}+(1-s) h_{T x} \int} f_{H}(d) d h d s
$$

$\eta$ defined in equation (7) is the obstacle thinning factor.

To ensure effective mode selection and successful communication in D2D pair in either microwave or mmWave, it is vital that the interference caused by and suffered by the D2D equipment is mitigated efficiently. Some of the research focused on hybrid D2D interference management have put forward mechanisms such as Fractional Frequency Reuse (FFR) technique (Eslami, Mirjalily, \& Davidson, 2020; Kim et al., 2018; Ningombam, et al., 2017) or a variant of FFR (Tabassum et al., 2020). Future network will have to coexist with microWave and mmWave band infrastructure side-by-side thereby necessitating the need for developing mechanisms that can accommodate seamless transitioning between both bands. Figure 2 depicts a 5G network architecture comprising microWave, mmWave and massive MIMO.

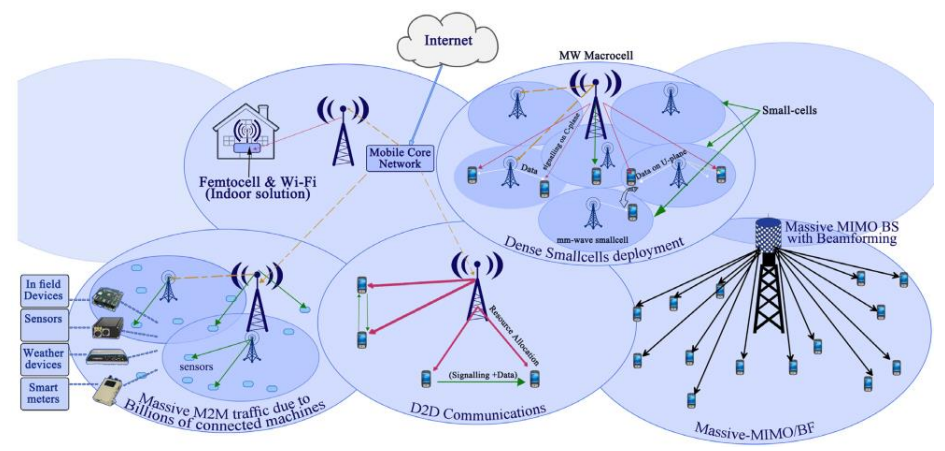

Fig. 2: 5G Network architecture comprising microWave and mmWave bands.

\section{Reviews on D2D Deployment in Hybrid Cellular Network Environment}

D2D as an emerging technology for spectrum efficiency and user experience improvement, has gained interest in both academia and communication industry as a promising solution. However, hybrid cellular network environment is the future target for real world application as mmWave is gradually deployed across the globe where current microWave band still exists and serve massive number of users. Therefore, some research effort focused on proffering mechanism for seamless deployment and utilization of D2D communication in this network scenario are reviewed in this section.

Li \& Tsai. (2018) reported to have designed an efficient methodology for establish and using D2D communication technology in a 5G network, comprising microWave and mmWave bands. The mechanism is based on both inband and outband techniques, leveraging on Wi-Fi direct protocol (Khan, Cherif, Filali, \& Hamila, 2017) for outband communication, while requiring a full eNB control for inband D2D communication (Hayat, Ngah, \& Zahedi, 2019). The 
authors proposed a two-phased approach, where phase one requires UE initiate request to communicate and the request is routed via the eNB and the Enveloped Pack Core (EPC), with confidential details of the requesting UE such as International Mobile Subscriber Identity (IMSI), location and distance to base station, the request is granted and controlled by the eNB if the identity, location and network constraints are met as judged by the EPC, for same cell devices or adjacent cell devices, otherwise, they are instructed to communicate via cellular network. The second phase of the hybrid mechanism is independent of the cellular network, here the intending source device sends request beacon to the intended destination and will transmit data only after a successful acknowledging beacon is received, in the event of failure, the beacon is resent at a predetermined interval for a set number of times before the communication attempt is abandoned as unreachable. The solution, though promising does not consider inherent mmWave network challenges like NLOS and beamforming.

Wang et al. (2019) presented hybrid communication model for mmWave and microWave band D2D communication underlaying 5G cellular system is proffered. The approach proposed, ensures the establishment of D2D link either via mmWave band when available and unobstructed or microwave band otherwise. The mmWave is only available via a line-ofsight (LOS) scenarios (Kakkavas et al., 2019), otherwise it is assumed blocked. To ensure hybrid D2D communication, BS is informed of D2D communication intention by the source UE, the BS locates and broadcast D2D-link-setup-request to the UEs, the DUE pair receives request and setup beam alignment process (Hassanieh et al., 2018) in the microWave band, DUEтx sends probing signals towards DUERx in a circular pattern, all received signals are recorded and compared and forwarded to the BS, the BS sends the information to DUETx to select the strongest signal received location for data transmission, in the case the received power does not reach pre-defined threshold, the data transmission between the DUE pair is performed via microwave.

Eslami et al. (2020) considered co-tier and cross-tier interference in deployment of D2D communication link in hybrid mmWave and microWave network infrastructure. To provide a solution, all devices are allocated to access the spectrum either in reuse or dedicated mode, the mixed integer nonlinear nonconvex problem (Mahajan, Leyffer, Linderoth, Luedtke, \& Munson, 2020) is solved based on problem decomposition method, the matching problem is addressed using KuhnMunkres algorithm (Chen, Tian, \& Wang, 2014). The authors report that the proposed strategy solves admission, power control problems optimally, including matching subproblems, by using heuristic suboptimal algorithm (Stern, Dreiman, \& Valenzano, 2019) to allocate free sub-channels. The solution for interference is based entirely on a Modified Fractional Frequency Reuse scheme (Tabassum, Islam, \& Kaiser, 2020) proffered by the authors, which is subject to minimum sum rate and transmission power constraints.
Fahim \& Samad. (2019) attempted to provide a combined mechanism for mmWave and microWave band cellular network for implementing D2D communication for both indoors and outdoors scenarios, while considering rain and fog attenuation effect on LOS (Elmutasim \& Mohd, 2021). In the work, the authors suggested band selection technique that is based on the Angle of Arrival (AoA) (Dong et al., 2020) Peaks from mmWave band to the intending D2D transmitter, whereby the threshold for inconsistency is measured and mmWave is either selected or rejected for establishing the D2D link. While microWave band is reserved as redundant link establishing band, while computing the SINR for the mmWave band, considering the attenuation due to rain and fog before final link establishment is performed (Semiari, Saad, \& Bennis, 2017). Kamruzzaman,et al. (2019) developed an algorithm based on resource allocation for multi-tier network considering a stochastic geometry, by first considering mode-selection option between D2D device or Cellular device, to either use cellular mode (CM) or switch to a sharing mode (SM) for D2D communication.

Bastos, Silva, and DaSilva. (2018) developed Network Assisted Routing (NAR), based on greedy heuristics to undertake resource allocation within the network, by defining multi-paths between the base station and D2D devices based on shortest path and channel quality indicator (CQI) within a set parameterized threshold. Chiu, Lin, Lin, and Wei. (2017) developed a multiuser D2D which has a systematic methodology that checks whether the antenna combination in a D2D network is capable of eliminating cross-pair interference and, thereby, ensuring interference-free concurrent transmissions. If the interference can be eliminated, then MD2D applies a bucket-based Degree of freedom, DoF assignment algorithm to determine an effective antenna usage configuration that handles the interference.

Doumiati, Assaad \& Artail. (2019) derived a scheme which divides the whole network into multiple groups, each served on a different frequency, and the interference within each group is managed by TIM, based only on the connectivity pattern and not on the instantaneous channel state information (CSI). They model TIM as a low-rankmatrix-completion problem (LRMC) problem and solve it using a novel and low-complex scheme based on semidefinite programming (SDP). As for the clustering part, we develop a clustering algorithm that is suited for the LRMC approach to solve TIM, while building on the SDP relaxation of the maximum-k-cut algorithm, and extending it to account for each cluster's capacity.

Some of the work by researchers in this field covers hybrid network scenarios, while most proposed solution from literature are focused on either microWave or mmWave only. However, not all the Hybrid solution cover all the challenges expected on the Hybrid network scenario, especially interference and mmWave band peculiarities such as NLOS conditions. Table 1 presents a summary of some of the reviewed papers. 


\begin{tabular}{|c|c|c|c|}
\hline $\begin{array}{c}\text { Authors } \\
\text { (Year) }\end{array}$ & Title & Proposed Solution & Challenges \\
\hline $\begin{array}{l}\text { Eslami et al., } \\
(2020)\end{array}$ & $\begin{array}{l}\text { Spectrum-Efficient QoS-Aware Resource } \\
\text { Assignment for FFR-Based D2D-Enabled } \\
\text { Heterogeneous Networks }\end{array}$ & $\begin{array}{l}\text { Presented a mode selection strategy for D2D link establishment using } \\
\text { either Dm or RM, interference mitigation is covered using a modified } \\
\text { Fractional frequency reuse mechanism. }\end{array}$ & No consideration for NLOS scenario in mmWave. \\
\hline $\begin{array}{l}\text { Fahim and } \\
\text { Samad, } \\
(2019)\end{array}$ & $\begin{array}{l}\text { Device-to-Device Communication with } \\
\text { Weather Considered Combined Mechanism } \\
\text { of Millimeter Wave and Microwave Band }\end{array}$ & $\begin{array}{l}\text { Proposed a hybrid band selection mechanism applicable to indoor } \\
\text { and outdoor transmission scenario, while considering weather effect. }\end{array}$ & Does not cover interference management. \\
\hline $\begin{array}{l}\text { Kamruzzam } \\
\text { an, Sarkar, } \\
\text { Gutierrez, } \\
\text { and Ray, } \\
(2019)\end{array}$ & $\begin{array}{l}\text { A Mode Selection Algorithm for Mitigating } \\
\text { Interference in D2D Enabled Next- } \\
\text { Generation Heterogeneous Cellular } \\
\text { Networks }\end{array}$ & $\begin{array}{l}\text { Proposed a mode selection algorithm, aimed at mitigating the } \\
\text { interference inherent in D2D enabled future generation heterogenous } \\
\text { networks. }\end{array}$ & $\begin{array}{l}\text { No significant improvement is achieved for long } \\
\text { distance D2D communication within the heterogenous } \\
\text { network. }\end{array}$ \\
\hline $\begin{array}{l}\text { Wang et al., } \\
(2019)\end{array}$ & $\begin{array}{l}\text { A hybrid communication model of } \\
\text { millimeter wave and microwave in D2D } \\
\text { network }\end{array}$ & $\begin{array}{l}\text { Developed a hybrid D2D link mechanism considering received } \\
\text { power threshold and LOS availability in choosing mmWave or } \\
\text { microWave band. }\end{array}$ & Interference mitigation strategy was not presented. \\
\hline $\begin{array}{l}\text { Doumiati, } \\
\text { Assaad, } \\
\text { Artail, } \\
(2019)\end{array}$ & $\begin{array}{l}\text { A Framework of Topological Interference } \\
\text { Management and Clustering for D2D } \\
\text { Networks }\end{array}$ & $\begin{array}{l}\text { They develop a joint clustering and } \\
\text { topological interference management (TIM) framework for a } \\
\text { Device-to-Device (D2D) network. }\end{array}$ & $\begin{array}{l}\text { The authors did not Consider the line of sight and } \\
\text { beam Alignment and its Negativities in the system. }\end{array}$ \\
\hline $\begin{array}{l}\text { Li and Tsai, } \\
(2018)\end{array}$ & $\begin{array}{l}\text { Design and Evaluation of a Hybrid D2D } \\
\text { Discovery Mechanism in 5G Cellular } \\
\text { Networks }\end{array}$ & $\begin{array}{l}\text { D2D communication using inband or outband techniques for } \\
\text { mmWave and microWave band. }\end{array}$ & $\begin{array}{l}\text { Does not consider peculiar mmWave challenges like } \\
\text { NLOS scenarios. }\end{array}$ \\
\hline $\begin{array}{ll}\text { Bastos, } \\
\text { Silva, and } \\
\text { Da Silva, } \\
(2018)\end{array}$ & $\begin{array}{l}\text { A combine mode selection based resource } \\
\text { allocation and interference control } \\
\text { technique for D2D communication }\end{array}$ & $\begin{array}{l}\text { Presented a Network Assisted Routing Algorithm for D2D } \\
\text { communication in 5G networks. }\end{array}$ & $\begin{array}{l}\text { The obvious limitation of the proposed technique is the } \\
\text { reliance on a fully function eNB with good coverage, } \\
\text { and no specific technique to limit the inherent Inter } \\
\text { Cell Interference imposed by the communicating D2D } \\
\text { pair sharing resource with the cellular devices within } \\
\text { the network. }\end{array}$ \\
\hline $\begin{array}{l}\text { Chiu, Lin, } \\
\text { Lin, and } \\
\text { Wei, }(2017)\end{array}$ & $\begin{array}{l}\text { Empowering Device-to-Device Networks } \\
\text { with } \\
\text { Cross-Link Interference Management }\end{array}$ & $\begin{array}{l}\text { This work presents a systematic MIMO interference } \\
\text { management scheme to handle interference in a } \\
\text { complex multiuser D2D network. }\end{array}$ & $\begin{array}{l}\text { The system operates only in } 4 \mathrm{G} \text { architecture not } 5 \mathrm{G} \text { or } \\
\text { Simulteneously }\end{array}$ \\
\hline
\end{tabular}




\section{Challenges of D2D Links in Hybrid Cellular Network}

Some of the major challenges that researchers aim to solve in order to effectively deploy D2D communication link in Hybrid Network environment for 5G and beyond are;

i. Mode Selection: The challenge of selecting the most reliable link establishment mode either Dedicated Mode or Reuse Mode for continuous link between communicating D2D pairs.

ii. Band Switching: In the case of mmWave and microWave availability, the choice of selecting, establishing and maintaining D2D link and the threshold requirement for switching in the result of degradation, interference or blockage.

iii. Blocking and Deafness: Challenges of setting threshold values for acceptable deafness in mmWave band communication and development of reliable NLOS correction technique to counter blockage.

iv. Interference: Developing a robust interference management mechanism applicable to both mmWave and microWave band underlaid with D2D to improve cellular user QoS and ensure reliable D2D communication.

Some of these challenges are being solved, however, they are mostly solved as stand-alone solutions to improve performance where needed, such as mode selection for mmWave and microWave band network presented by (Feng et al., 2019) does not consider D2D communication link, likewise the work presented by (Moltafet, Mokari, Joda, Sabagh, \& Zorzi, 2018) also suggest dual mode select scheme but does not consider D2D links, for band switching some promising research effort like the work by (Semiari et al., 2017).

\section{ConCLUSIONS}

D2D has been established to be beneficial technology in current and future communication network, however, there are challenges associated widespread adaptation in current cellular network. As a solution to future network spectrum scarcity and improved user service experience and quality, research efforts are geared toward developing mechanism for enabling D2D to coexist in hybrid mmWave and microWave cellular network bringing the benefits of D2D to both bands while also providing solution to mitigate interference and overcome inherent challenges in mmWave band that limits D2D utilization. This paper provided some key features of the D2D technology, state-of-the-art in the research field of D2D in hybrid bands and challenges being encountered.

\section{REFERENCE}

Asadi, A., Wang, Q., \& Mancuso, V. (2014). A survey on device-todevice communication in cellular networks. IEEE Communications Surveys Tutorials, 16(4), 1801-1819.

Bastos, A. V. Silva, C. M. \& Da-Silver-Jnr, D. C. (2018). Assisted Routing Algorithm for D2D Communication in 5G Wireless Networks. IEEE 2018 wireless days, 28-30

Chen, N., Tian, H., \& Wang, Z. (2014). Resource allocation for intracluster D2D communications based on Kuhn-Munkres algorithm. Paper presented at the 2014 IEEE 80th Vehicular Technology Conference (VTC2014-Fall).

Chevillon, R., Andrieux, G., Négrier, R., \& Diouris, J. F. (2018). Effects of directional antennas on outband D2D mmWave communications in heterogeneous networks. AEU - International Journal of Electronics and Communications, 96, 58-65. doi:10.1016/j.aeue.2018.09.006

Chiu, S. Lin, K. C. Lin, G. \& Wei, H. (2017) Empowering Device-toDevice Networks with Cross-Link Interference Management, IEEE Transactions on Mobile Computing. DOI 10.1109/TMC.2016.2582169.

Dai, X., \& Gui, J. (2020). Joint access and backhaul resource allocation for D2D-assisted dense mmWave cellular networks. Computer Networks, 183, 107602. doi:10.1016/j.comnet.2020.107602

Doumiati, S. Assaad, M. \& Artail, H. A. (2019). A Framework of Topological Interference Management and Clustering for D2D Networks. IEEE International Workshop on Computer-Aided Modeling Analysis and Design of Communication Links and Networks, IEEE CAMAD, DOI 10.1109/TCOMM.2019.2931319

Elmutasim, I. E., \& Mohd, I. I. (2021). Examination Rain and Fog Attenuation for Path Loss Prediction in Millimeter Wave Range. Paper presented at the Proceedings of the 11th National Technical Seminar on Unmanned System Technology 2019.

Eslami, L., Mirjalily, G., \& Davidson, T. N. (2020). Spectrum-Efficient QoS-Aware Resource Assignment for FFR-Based D2D-Enabled Heterogeneous Networks. IEEE Access, 8, 218186-218198. doi:10.1109/ACCESS.2020.3041770

Fahim, A. A. R., \& Samad, M. F. (2019). Device-to-Device Communication with Weather Considered Combined Mechanism of Millimeter Wave and Microwave Band. 2019 4th International Conference on Electrical Information and Communication Technology, EICT 2019, 1-6. doi:10.1109/EICT48899.2019.9068843

Hayat, O., Ngah, R., \& Zahedi, Y. (2019). In-band device to device (D2D) communication and device discovery: A survey. Wireless Personal Communications, 106(2), 451-472.

Höyhtyä, M., Apilo, O., \& Lasanen, M. (2018). Review of latest advances in 3GPP standardization: D2D communication in 5G systems and its energy consumption models. Future Internet, 10(1), 3.

Hussain, N., Jangsher, S., Arafah, M., Mao, Y., \& Li, V. O. K. (2019). Joint Relay-Spectrum Selection in Hybrid Millimeter-Microwave Cooperative (HMMC) Network Using Fall-Back Approach. IEEE Access, 7, 63089-63098. doi:10.1109/ACCESS.2019.2917270

Kakkavas, A., Seco-Granados, G., Wymeersch, H., Garcia, M. H. C., Stirling-Gallacher, R. A., \& Nossek, J. A. (2019). 5G downlink multi-beam signal design for LOS positioning. Paper presented at the 2019 IEEE Global Communications Conference (GLOBECOM).

Kamruzzaman, M. D. Sarkar, N. I. \& Ray, S. K. (2019). A mode Selection algorithm for mitigating interference in D2D enabled next-generation heterogenous cellular networks. IEEE (ICOIN 2019), 131-135

Kim, J., Kim, T., Noh, J., \& Cho, S. (2018). Fractional frequency reuse scheme for device to device communication underlaying cellular on wireless multimedia sensor networks. Sensors (Switzerland), 18. doi:10.3390/s18082661 
Kuruvatti, N. P., Hernandez, R., \& Schotten, H. D. (2019). Interference Aware Power Management in D2D Underlay Cellular Networks. 2019 IEEE AFRICON.

Li, M., \& Tsai, H. L. (2018). Design and Evaluation of a Hybrid D2D Discovery Mechanism in 5G Cellular Networks. International Conference on Ubiquitous and Future Networks, ICUFN, 2018-July, 241-243. doi:10.1109/ICUFN.2018.8436771

Lien, S.-Y., Chien, C.-C., Tseng, F.-M., \& Ho, T.-C. (2016). 3GPP device-to-device communications for beyond $4 \mathrm{G}$ cellular networks. IEEE Communications Magazine, 54(3), 29-35.

Mahajan, A., Leyffer, S., Linderoth, J., Luedtke, J., \& Munson, T. (2020). Minotaur: A mixed-integer nonlinear optimization toolkit. Mathematical Programming Computation, 1-38.

Moltafet, M., Mokari, N., Joda, R., Sabagh, M. R., \& Zorzi, M. (2018). Joint access and fronthaul resource allocation in dual connectivity and CoMP based networks. Paper presented at the 2018 IEEE International Conference on Communications (ICC).

Ningombam, D. D., Pyun, J. Y., Hwang, S. S., \& Shin, S. (2017). Fractional frequency reuse scheme for interference mitigation in device-to-device communication underlying LTE-A networks. 2017 51st Asilomar Conference on Signals, Systems, and Computers.

Noura, M., \& Nordin, R. (2016). A survey on interference management for Device-to-Device (D2D) communication and its challenges in 5G networks. Journal of Network and Computer Applications, 71, 130-150. doi:10.1016/j.jnca.2016.04.021

Nuerxiati, N., Zhonggui, M., Xingye W., (2019). Modeling and PerformanceOptimization of Heterogeneous Cellular and D2D Networks. 28 ${ }^{\text {th }}$ Wireless and Optical Communications Conference (WOCC), 2019

Prado, G. M. M., Cardieri, P., \& Brito, J. M. C. (2020). Packet Retransmission in Hybrid Millimeter-Wave and Microwave D2D Communication System. Wireless Personal Communications, 110, 1251-1270. doi:10.1007/s11277-019-06784-2

Qiao, J., Shen, X., Mark, J., Shen, Q., He, Y., \& Lei, L. (2015). Enabling device-to-device communications in millimeter-wave 5G cellular networks. IEEE Communications Magazine, 53, 209-215. doi:10.1109/MCOM.2015.7010536

Roslee, M., Nizam, F., \& Yusoff, Z. (2020). Algorithm and Scheme for D2D Communication in $4 \mathrm{G} / 5 \mathrm{G}$ Networks. 2020 International Conference on Electrical, Communication, and Computer Engineering (ICECCE), 1-6. doi:10.1109/ICECCE49384.2020.9179396

Sanusi, I. O., Nasr, K. M., \& Moessner, K. (2019). Channel assignment and power control for D2D-enabled cellular networks. Proceedings - 2019 International Conference on Computing, Electronics and Communications Engineering, iCCECE 2019, 225228. doi:10.1109/iCCECE46942.2019.8941981

Sawsan, S., \& Ridha, B. (2021). Joint uplink and downlink resource allocation for device to device communications", IET Communications

Semiari, O., Saad, W., \& Bennis, M. (2017). Joint millimeter wave and microwave resources allocation in cellular networks with dualmode base stations. IEEE Transactions on wireless communications, 16(7), 4802-4816.

Song, L., Niyato, D., Han, Z., \& Hossain, E. (2015). Wireless device-todevice communications and networks: Cambridge University Press.

Stern, R., Dreiman, G., \& Valenzano, R. (2019). Probably bounded suboptimal heuristic search. Artificial Intelligence, 267, 39-57.

Tabassum, F., Islam, A. K. M. N. M. N., \& Kaiser, M. S. (2020). Modified Fractional Frequency Reuse Scheme for Nonorthogonal Multiple Access Networks. 2020 Third International Conference on Smart Systems and Inventive Technology (ICSSIT).

Wang, F., Wang, H., Feng, H., \& Xu, X. (2019). A hybrid communication model of millimeter wave and microwave in D2D network. IEEE Vehicular Technology Conference, 2016-July, 15. doi:10.1109/VTCSpring.2016.7504074
Wu, S., Atat, R., Mastronarde, N., \& Liu, L. (2018). Improving the Coverage and Spectral Efficiency of Millimeter-Wave Cellular Networks Using Device-to-Device Relays. IEEE transactions on Communications, 66, doi:10.1109/TCOMM.2017.2787990
2251-2265. 